\section{Tendencies to eye movement, and misperception of curvature, direction, and length*}

\author{
VEIJO VIRSU† \\ University of Helsinki, Helsinki, Finland
}

From previous studies of eye movements, three types of eye-movement tendency can be inferred: (1) tendency to rectilinear eye movements, (2) tendency to horizontal or vertical eye movements, and (3) tendency to center-of-gravity fixations. The possible influence of these eye-movement tendencies on perception was investigated in two experiments. In Experiment 1, errors in perceived location of intersection in arc figures were studied varying arc-point distance and arc length. Tendencies 1 and 2 accounted very well for the resultant S-shaped functions. In Experiment 2, the Müller-Lyer illusion with three different oblique angles and a line-segment illusion were measured as a function of the distance between the vertex and the center of gravity of the arrowhead. Tendency 3 accounted well for the inverted- $U$ forms of the obtained functions but not for the increase of error with increasing angle.

One view concerning interaction between movements and perception is that the neural state which initiates movements determines conscious experience of perception at least partially. Recently this notion has been advocated by Festinger, Burnham, Ono, and Bamber (1967), Festinger, White, and Allyn (1968), and Grovitz and Daves (1962). A difficulty of the notion is to refute the conventional view that perception determines movements and tendencies to movement, and not vice versa. Yet this may not be an overwhelming difficulty, for there already are results suggesting that in some cases conscious perception does not precede movement and hence cannot be the cause of the movement (Kahneman, 1967). For example, simple reaction time to light is about the same as the delay of visual experience (Mayzner, Tresselt, \& Helfer, 1967), and movements initiated by a visual stimulus can occur even though the stimulus triggering the response does not evoke a visual experience (Fehrer \& Raab, 1962). Tendency to movement, however, could precede and determine both movements and perception in these cases.

There is a small amount of direct evidence that tendency to movement (or efferent readiness, as it is called by Festinger) influences perception. The results of four experiments by Festinger et al (1967) showed predicted modification in visual perception of curvature as a result

*Supported by Kamarineuvos H. Rosenberg's Travel Grant from University of Helsinki. The study was prepared during the author's visit at Stanford University and the University of Michigan. I am grateful to D. J. Weintraub and D. H. Krantz for comments, criticism, and the use of their research facilities.

†Address: Department of Psychology, University of Helsinki, Helsinki 17, Finland. of new afferent-efferent associations learned during the experiments. The data are not readily interpreted without assuming changes in efferent readiness causing the modification of perception, because control Ss having similar conditions as experimental $S s$ but not learning new afference-efference relationships showed significantly less modification than did the experimental Ss.

Assuming that efferent readiness determines perception, one should be able to predict not only perceptual modifications during experiments but also how an $\mathrm{O}$ will perceive a given stimulus when he enters the experiment, that is, one should be able to predict perceptual outcomes from characteristics of the stimuli to be perceived. If this were possible, efferent readiness theory of perception could be used, for instance, to explain geometrical illusions. Two experiments were designed to study this possibility.

\section{EXPERIMENT 1}

It is known that eye movements are inaccurate in tracking curved or tilted contours, or objects performing curvilinear motions. Stroud (reported by Whitteridge, 1960, pp. 1103-1104) recorded pursuit eye movements when an $\mathrm{O}$ followed a spot performing a simple curvilinear motion. At first the eyes of the $\mathrm{O}$ attempted to follow the sinusoidal movement by rectilinear horizontal or vertical saccadic movements. After a few repetitions the movements became oblique even though they remained curvilinear. With prolonged practice the movements became curvilinear and very accurate. Similar results were found by Dodge (1907) when Os followed the movement of a pendulum.

Stration (1902) recorded eye movements by $O S$ who tracked the contours of circles. He found that the saccades did not follow the appropriate curvilinear paths but formed straight lines. Yarbus (1967, pp. 140-141) reported that saccades in a horizontal or vertical direction are in most cases recorded on stationary photosensitive paper as straight lines, but saccades performed at an angle to these two directions are most frequently recorded as curved lines. A figure presented by Yarbus suggests that the curvedness results from an initial attempt to follow a tilted line horizontally or vertically, and from a correction in the direction of the eye movements toward the veridical location of the line when the line is lost from the fovea (or maybe from an intended spot inside the fovea) as a result of the wrong initial tracking direction.

The results show that unpracticed eye movements for curved or tilted paths are inaccurate. The inaccuracy does not result from defects of the muscular system, since eventually the movements can be accurate. The inaccuracy is not caused by nonveridical visual experiences either, because a circle, for instance, is perceived as a perfect circle and not as an irregular straight-line pattern that would correspond to the eye movements. ${ }^{1}$ Hence, the tracking errors probably result from inappropriate movement tendencies or inappropriate efferent readinesses activated by the stimuli to be tracked.

The movement records suggest that there is an inappropriate efferent readiness for horizontal and vertical eye movements. Assuming that a decision mechanism "switches on" either a horizontal or a vertical movement tendency, depending on which one would give better fit in tracking, one can expect that a line oriented nearly horizontally evokes (1) readiness to perform horizontal eye movements, (2) nearly horizontal tracking eye movements (not completely horizontal because of corrections based on afferent feedback), and (3) perceptual inclination of the line toward the horizontal, if movement tendencies influence perception. Corresponding events should occur with a nearly vertical line. Somewhere between the vertical and horizontal directions, the opposite inclination tendencies should cancel each other. Assuming that the indifference direction is a middle position between the horizontal and vertical, as a rough but economical generalization one could expect that the amount of perceptual inclination error $B$ is

$$
B=b_{1} \sin 4 A,
$$

where $b_{1}$ is a parameter and $A$ the deviation of the direction of the line from the vertical (or horizontal). This function 


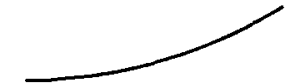

Fig. 1. The converging-arcs illusion; the location of intersection is misperceived.

could be useful as a preliminary approximation, because it gives a required zero error not only between the two cardinal directions but also when a line is oriented horizontally or vertically; as a line has these orientations, horizontal and vertical movement tendencies are adequate and no error should occur.

There is some evidence supporting Eq. 1 as an approximation of the basic function in perceptual tilt errors. In two experiments by Weintraub and Virsu (1971), Os estimated the location of intersection in a display formed by two converging straight-line segments. The errors in apparent location of the intersection, if considered as errors in perceived inclinations of the lines, could be characterized by Eq. 1, except that the errors were larger around the horizontal direction than around the vertical and, partially because of this, the zero intercepts were displaced from those implied by Eq. 1. Still the main course of the tilt error for inclinations $A$ from -84 to $+169 \mathrm{deg}$ showed the variation and maxima in agreement with Eq. 1. Similar error functions were obtained also by Obonai (1931) for the perceptual inclination of a line segment pointing toward a point on another line segment, and by Hofmann and Bielschowsky (1909) for inclinations of the apparent horizontal in a field of tilted lines. ${ }^{2}$

Besides suggesting a readiness for horizontal and vertical eye movements, the eye-movement records suggest a tendency to rectilinear eye movements in tracking of curvilinear contours and motions. This tendency would lead one to expect underestimation of curvature of arcs, which are so short or so little curved that they can be tracked without performing rectangular corrections to the eye movements (a more detailed discussion on this is presented by $\mathrm{Virsu}^{3}$ ).

The arc figure in Fig. 1 was devised to test the ideas presented above. The dot is the geometrically correct lower intersection of the arcs but it seems to be too high. This can be a joint effect of curvature underestimation and inclination of the tracked continuations of the arcs. The interaction of these two sources of error is studied in the experiment below. As tracking of the arcs during the experiment provides afferent feedback information about the true curvatures of the arcs and is likely to induce corrections in movement tendencies (Festinger, White, \& Allyn, 1968), the illusion should diminish during the experiment. A simple check of this was included in the design.

\section{Method}

Subjects. Twenty students, 5 women and 15 men, from different departments at Stanford University volunteered as Ss.

Stimuli. Ten different versions of Fig. 1 with the intersection point omitted were drawn with black India ink on $14 \times 21.5 \mathrm{~cm}$ sheets of paper. Line thickness was $0.3 \mathrm{~mm}$. In all figures, the basic pattern from which the two arcs were taken consisted of two circles the radii of which were $44.6 \mathrm{~mm}$. Therefore, the curvatures of all the arcs were the same $.22 \mathrm{~cm}^{-1}$ (curvature is the inverse of the radius of curvature). The distance between the centers of the circles was $87.8 \mathrm{~mm}$. Hence, the basic circles intersected at two points, at the distance of $15.5 \mathrm{~mm}$ or $20 \mathrm{deg}$ (of the center angle) from each other. The midpoint of the basic configuration was placed near the middle of the sheets so that the lower intersection point of the circles was always about $8 \mathrm{~mm}$ below the sheet middle. The two arcs were taken from the basic circles so that the angular distances, D, between the lower intersection point and the nearer arc ends were $18,36,54,72$, and $90 \mathrm{deg}$. For every $D$ there were two different arc lengths, $L$, 30 and $90 \mathrm{deg}$. Both $\mathrm{D}$ and $\mathrm{L}$ are measured as corresponding center angles of the basic circles.

Procedure. Each S was presented with a separate set of 11 stimulus figures. Ten irst figures consisted of the 10 variations xplained above, and the presentation order of these figures was independently andomized for each $S$. The 11th figure was the same as the first figure in the series.

The Ss were asked to estimate the location of the lower intersection point of the circular continuations of the arcs and to indicate its place by marking a small point on the stimulus display sheet with India ink. As it is possible that hand movements bias this estimate, the Ss were asked to look carefully at the figure resulting from the point and the arcs, and to mark a second point to indicate the final judgment. The first point was black and the second red. The figures were presented one at a time at about $38 \mathrm{~cm}$ distance from $S$ 's eyes. The sheets were presented on a table so that the intersection pointed away from $S$ and the symmetry axis of the figure was vertical with respect to S's visual field. Tracking of the continuations with hand movements and tilting of the sheets were not allowed, but eye movements were free.

\section{Results}

The center line of the stimulus display is the vertical line that includes the two intersection points of the basic circles. The 440 estimates collected were measured along the center line as deviations from the true lower intersection. If a marked intersection was above the true intersection it was scaled negative, and if it was under the true intersection it was scaled positive. These measurements, as well as all the later measurements pertaining to the amount of error, are expressed in degrees of the corresponding center angles of the basic circles. All the estimates were projected onto the center line, because there were no systematic lateral errors. The differences between the first and second markings were also unsystematic and not statistically significant. Therefore, these two estimates were averaged in each figure to form one single measurement.

Figure 2 shows the mean results of the 20 Ss. The open and filled-in circles present the means obtained with 30- and 90-deg arcs, respectively. The smooth curves are theoretical functions explained later. One standard error of the mean is depicted on either side of the mean by a vertical bar. At its maximum, the new illusion was very powerful. With 30-deg arc and 72-deg distance, the error mean deviates 2.4 standard deviations from the true value, the lower intersection point being estimated nearly 1 in. too low in this figure. A repeated-measurements analysis of variance resulted $F=32.3(\mathrm{df}=4 / 76)$ for the five distances, $D, F=37.1$ ( $\mathrm{df}=1 / 19$ ) for the two arc lengths, $\mathrm{L}$, and 


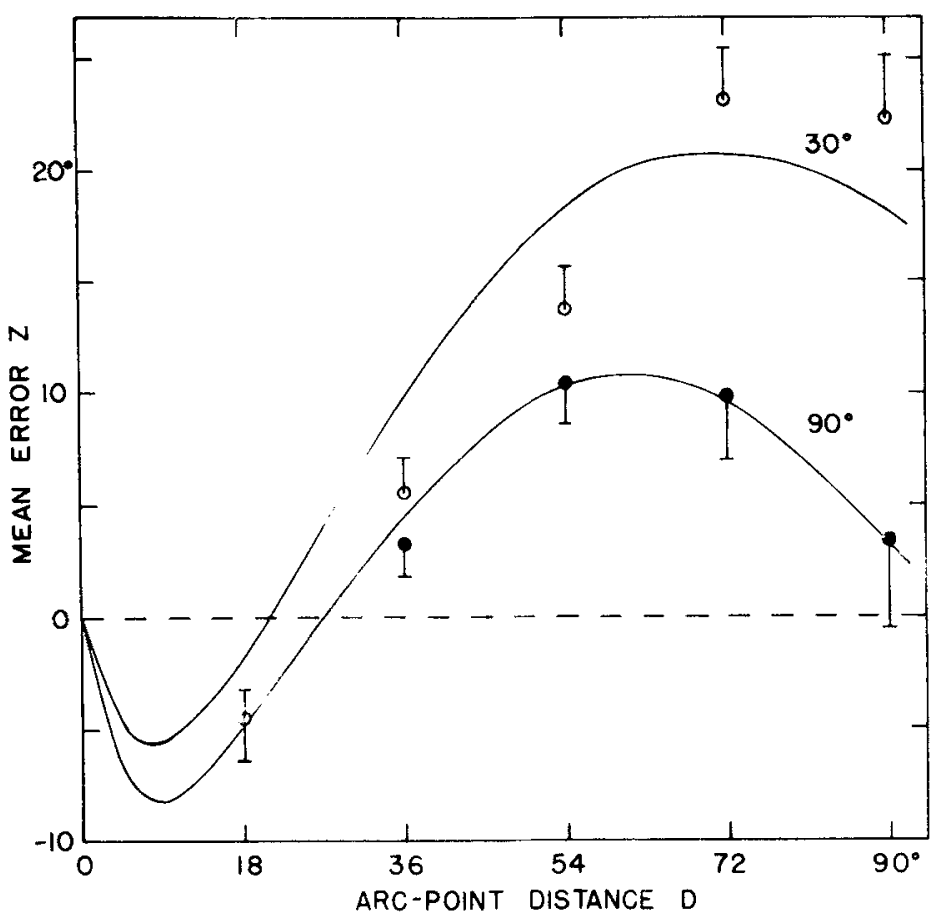

Fig. 2. The misperception of the location of intersection as a function of arc length ( 30 and $90 \mathrm{deg}$ ) and the distance between the intersection and the arcs. The units of measurement are degrees of center angle.

$F=12.0(\mathrm{df}=4 / 76)$ for the interaction of $\mathrm{D}$ by $\mathrm{L}$. Thus, the differences in the main effects as well as their interaction were all statistically highly significant.

Comparisons of the 1st figure estimates with the 11th figure estimates (the 1st figure repeated) showed that the amount of illusion diminished with practice. The estimates of $15 \mathrm{Ss}$ became more veridical in the course of the estimation of 10 different arc figures. $A$ t test for correlated observations was computed for the differences of the absolute values of the two estimates. It resulted in a significant one-sided $t$ value $(t=1.95, \quad d f=19$, $\mathrm{p}<.05$ ). This result would be predicted if afferent feedback obtained from eye movements led to decrement of the illusion.

\section{Analysis of Results}

It was assumed that the perceptual displacement of intersection in Fig. 1 was a joint effect of two error-producing factors, one of which was the underestimation of curvature induced by the tendency to perform rectilinear eye movements and the other the perceptual inclination of the continuations of the arcs induced by the tendency to perform horizontal or vertical eye movements. Let us denote the former error component by $x$ and the latter by $y$, both understood in terms of a perceptual displacement of the lower intersection along the center line of the display. For simplicity, let us assume that $\mathrm{x}$ and $\mathrm{y}$ are combined additively in the total displacement $z$ measured in the experiment. In order to evaluate the adequacy of the tendency-to-movement explanation as an explanation of the present results, total error $z$ must be analyzed to the two component errors, $x$ and $y$. Rather lengthy elementary trigonometric calculations are necessary for the analysis, and therefore only the results of the calculations can be presented here.

Let us denote the true radius of curvature of the arcs used in the experiment by r. Misperception of curvature can be conceived as a change, $b_{0}$, of the radius of curvature; if the curvatures of the arcs are underestimated the apparent radius of curvature of each arc is $r+b_{0}$ $\left(b_{o}>0\right)$. If Ss track the continuations of the arcs according to the extended radius of curvature, a perceptual downwards displacement of the lower intersection results. This produces the error component $x$. Assuming that the middles of the arcs are not displaced in perception, it can be shown that

$$
\begin{aligned}
x= & \overline{\operatorname{arc}} \tan \left\{( r \operatorname { c o s } K ) ^ { - 1 } \left[\left(\left(r+b_{o}\right)^{2}\right.\right.\right. \\
& \left.-\left(r \cos K+b_{0} \cos (d+K)\right)^{2}\right)^{1 / 2} \\
& \left.\left.-b_{o} \sin (d+K)\right]\right\}-K,
\end{aligned}
$$

where $\mathrm{x}$ is the center angle corresponding to the displacement in the location of the intersection, $K$ the center angle between the lower intersection point and the horizontal line connecting the centers of the basic circles ( $K=10 \mathrm{deg}$ in the present case), and $d=D+1 / 2 L$, the angular distance between the middle of an arc and the lower intersection.

Error component y can be computed using inclination factor $B$ from Eq. 1. However, argument $\mathrm{A}$ of Eq. 1 must be redefined for arcs, because in Eq. 1 it was defined for straight lines (as the deviation of the direction of a line from the vertical). This definition is not applicable to arcs since arcs do not possess the required linear direction. A definition that is more useful with arcs and is not at variance with the earlier definition is that $A$ is the deviation of the intended tracking direction from the vertical. The definition is based on the assumption that the direction which is actually misperceived in an experiment is the direction estimated by an $\mathrm{O}$, that is, both $\mathrm{A}$ and $\mathrm{B}$ are determined on the basis of the tracking task given in the experiment.

Since the continuations of the arcs were tracked in the present experiment, inclination factor B should be determined regarding the directions of the continuations. From the tendency to perform rectilinear eye movements, one could conclude that when an $O$ tracks the continuations of an arc he tends to follow the direction of the tangent at the end point of the arc. This tangent forms the best available substitute for the imagined continuation of the arc and its direction. As a result of the tendency to perform horizontal or vertical eye movements, the direction of the tangent and thus of the continuation is perceptually tilted at angle $B$. The effect of this tilt on the perceived location of the lower intersection (as the center angle of the basic circles) is then

$y=$

$$
\begin{aligned}
& \operatorname{arc} \tan \frac{(\cos A-\cos K) \cot (A+B)+\sin A}{\cos K} \\
& -\overline{\operatorname{arc} t a n} \frac{(\cos A-\cos K) \cot A+\sin A}{\cos K}
\end{aligned}
$$

where $\mathrm{K}$ is as before, $\mathrm{B}$ as in Eq. 1 , and $A=D+K$ the angle between the tangent at the end point of an arc and the visual field vertical (the center line in the present case).

Assuming that the total error is $z=x+y$, the least-square estimates for parameters $b_{0}$ and $b_{1}$ were approximated by iteration. With $30-\mathrm{deg}$ arcs the parameter values obtained were $b_{0}=9.0 \mathrm{~mm}$ and $b_{1}=-11.0 \mathrm{deg}$, and with 
90-deg arcs the values were $b_{0}=0.9 \mathrm{~mm}$ and $b_{1}=-11.8 \mathrm{deg}$. The smooth curves in Fig. 2 show the theoretical functions for $z$ with these parameter values. The fit is very good for 90-deg arcs, but it should be better for 30-deg arcs. However, the theoretical functions explain $92 \%$ of the total variation of the means, which shows that the two factors, underestimation of curvature and perceptual tilt of the continuations of the arcs, account very well for the data.

The values of parameter $b_{1}$ reflect the amount of perceptual tilt of the continuations of the arcs. The estimated values of $b_{1}$ are approximately the same for both arc lengths showing similar amounts of tilt in either case. This could have been expected, because there are no theoretical reasons to assume interaction between arc length and inclination of the continuations, if the inclination is caused by efferent readiness to perform horizontal or vertical eye movements.

It is presumed in the present calculations that there is no interaction between the tendency to rectilinear eye movements and the tendency to horizontal eye movements, that is, it is presumed that parameter $b_{o}$ remains constant in all orientations of the arcs. This may be a slight oversimplification, for it is possible that when an arc is oriented horizontally or vertically the tendency to horizontal or vertical eye movements strengthens the tendency to rectilinear tracking, thus increasing underestimation of curvature. The results suggest the existence of this interaction, for (1) the difficulty to obtain a good fit occurs with 30-deg arcs in which the underestimation of curvature plays a major role, and (2) the difficulty to obtain a good fit is mainly caused by the unexpectedly large errors with nearly horizontal arcs $(D=72$ and $90 \mathrm{deg}$ in $\mathrm{L}=30 \mathrm{deg}$ ). Later experiments, ${ }^{3}$ in which apparent curvatures of arcs were compared with those of circles, supported this interpretation, too, for more underestimation of the curvatures of short arcs was found with vertical arcs than with tilted arcs. The interaction was, however, weak and appeared only with the shortest arcs used in the experiments.

As parameter $b_{0}$ corresponds to the amount of misperception in the radius of curvature, its values can be compared directly with the curvature data of the experiments mentioned above. Value $b_{o}=9.0 \mathrm{~mm}$ implies $17 \%$ average underestimation of the curvatures of the 30-deg arcs, and value $b_{o}=0.9 \mathrm{~mm}$ implies $2 \%$ average underestimation of the curvatures of the 90-deg arcs of the present experiment. The respective curvature-underestimation values, interpolated from the data of the later experiments and corrected for the overestimation of size of open figures, were $16 \%$ and $3 \%$. These values are consistent with the values obtained as the values of parameter $b_{0}$, confirming the interpretation that underestimation of curvature is a factor in the illusion of Fig. 1.

\section{EXPERIMENT 2}

Festinger, White, and Allyn (1968) reasoned that the obliques in the Müller-Lyer figure bring into readiness for use inappropriate efferent programs causing the illusion. Their eye-movement data confirmed the old results according to which the illusion figure evokes inaccurate eye movements that are consistent with the illusion. In addition, their results showed that over- or undershooting of the test line induced small corrective saccades into the eye movements and that the decrement of the illusion with prolonged inspection was accompanied by an increase in the accuracy of eye movements. If eye movements were restricted by fixation, little or no decrement of illusion or change in eye movements occurred with repeated presentations. All these results can be predicted from the notion that inappropriate movement tendencies or efferent readinesses produce the Müller-Lyer illusion.

The efferent-readiness explanation of the Müller-Lyer illusion was judged incorrect by McLaughlin, DeSisto, and Kelly (1969). They referred to a study of parametric adjustments of eye movements (McLaughlin, Kelly, Anderson, \& Wenz, 1968) and stated that "the independent variable in this experiment was what Festinger would call 'efferent readiness,' and it turned out to have no effect whatever on perception." This strong conclusion, however, is not justified on the basis of the data. (1) The number of $S s$ in the experiment was three. It is quite impossible to base a "no-difference"

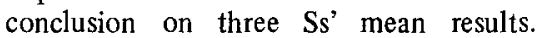
(2) There was a difference in the pointed locations of the targets (13.83 deg pretest vs $13.45 \mathrm{deg}$ posttest mean). The direction of the difference was the one that would be expected if efferent readiness had an effect on perception, but with the three $S$ s the difference was not statistically significant. (3) The number of practice fixations was 11. The speed of perceptual adjustment should have been extremely fast in order to produce significant perceptual changes under these conditions. The 11 practice fixations produced, however, a large adjustment in eye movements, suggesting that adjustments in eye movements take place faster than adjustments in perception. This is what must happen if tendencies to movement influence perception; tendencies to movement (inferred from actual movements) must change before perception can change. If perception, not movements, changed first, then obviously perception would determine movement tendencies and not contrariwise. (4) The pointer settings contained large errors regarding the true positions of the targets; for example, the mean setting for the 5-deg target was $9.37 \mathrm{deg}$, indicating $4.37-\mathrm{deg}$ mean error. Any interaction between perceptual adjustment and this kind of pointer-setting error could have masked a significant perceptual change (the total parametric adjustment of the eye movements was $3.63 \mathrm{deg}$, which is the upper limit of the expected perceptual change). (5) Presumably the three Ss were aware of the details of the experiment and of the physically true locations of the targets in postpractice settings. Therefore, the expected perceptual change should have occurred toward known nonveridicality of perception. However, cognitive and instructional effects can prevent perceptual changes during prolonged inspection of the Müller-Lyer figure (Mountjoy, 1965; Day, 1962); hence, the knowledge of the true target positions could have inhibited the possible perceptual changes caused by the parametric adjustment. In addition, the knowledge of the target positions could have contributed to the fast adjustment of the eye movements. (6) It could be added also that if no perceptual change whatever really occurred, the statement that "there is no evidence available to refute the conventional view that it is the perception that determines the eye movement [McLaughlin et al, 1969]" is incorrect, because the authors claim they recorded changes in eye movements that were not preceded or accompanied by respective changes in perception of location.

The evidence presented by McLaughlin et al is not strong enough for discarding the efferent-readiness explanation of the Müller-Lyer illusion. Festinger, White, and Allyn, however, did not explain how the oblique lines in the illusion figure could activate inappropriate efferent readinesses causing the illusion. Later, Kaufman and Richards (1969) and Richards and Kaufman (1969) published results that may form a basis for a more detailed and general explanation of the Müller-Lyer illusion. This possibility is investigated in the present experiment.

Kaufman and Richards found that, when viewing simple patterns like angles, triangles, squares, circles, line segments, dot patterns, etc., Ss tend to fixate near 
the center of gravity of the figure, provided that the figure subtends less than $5 \mathrm{deg}$ of visual angle. Even when Ss think that they are fixating corners of the figures, they may actually be fixating near the middle of the figure. This spontaneous fixation tendency cannot result from the visual experience of the Müller-Lyer illusion because it can be demonstrated with various patterns independently of the illusion figure and without length estimations. Now, if perceived length is even partially determined by anticipated amplitude of eye movements and if Ss are unwittingly anticipating scanning from one arrowhead center of gravity to another in the Müller-Lyer figure, the length illusion results. This must happen whatever geometric figures are placed at the ends of a neutral distance or a test line-the only requirement probably is that the figure at the end of the distance activates an unambiguous center-of-gravity tendency which is not nullified by any opposite tendency based on afferent feedback from corrective eye movements or competing centers of gravity. The many different versions of the Müller-Lyer figure presented in illusion literature show that a Müller-Lyer type of illusion, indeed, can be produced when squares, rectangles, triangles in any position, circles, arcs, straight line segments, etc., are placed at the ends of a test line. It is possible that also the circle illusions of Titchener and Delboeuf belong to the Müller-Lyer type of illusion; in these, the diameter of a circle corresponds to the test line and the inducing portion evoking center-of-gravity fixations is formed of arcs or circles. 4

The results by Kaufman and Richards (1969) for circles suggest that when the size of a circle increases, the scatter in fixations as well as differences between individuai $\mathrm{Ss}$ ' mean fixation positions increase, and the mean fixation positions become more and more off-centered. Off-centering begins with figures subtending less than $3 \mathrm{deg}$ of visual angle. Generalizing these results to fixation tendencies for inducing parts in the Müller-Lyer type of illusion, we could outline the following model for the illusion.

Let us denote the distance between the end of the test line and the geometric center of gravity of the adjacent inducing figure by CGD (center-of-gravity distance). An $O$ intends to fixate the end of the test line but it is postulated that actually he fixates toward the center of gravity of the inducing figure. If the estimates of the length of the test line were solely based on the peripheral anticipation to perform eye movements from one center of gravity to another in the inducing parts of a
Müller-Lyer-type figure, the error made by the $\mathrm{O}$ would be 2 CGD. However, when the CGD is large enough, over- or undershooting regarding the intended point of fixation is followed by a corrective flick toward the intended point of fixation (Festinger, White, \& Allyn, 1968). Thus, if eye movements are allowed, there is afferent feedback that reveals the tendency to center-of-gravity fixations inadequate for successful fixation toward the intended point of fixation. Because eye movements change as a result of this afferent feedback, eye-movement tendencies must change also as a result of afferent feedback, and the change takes place toward more veridical eye movements, eye-movement tendencies, and perception. Hence, afferent feedback obtained both during an experiment and before the experiment when scanning environmental objects limits the error to less than 2 CGD.

If CGD is small, the amount of afferent feedback revealing a discrepancy between the intended and actual fixation positions is small or negligible. Therefore, when CGD increases from zero, at first the amount of illusion is nearly 2 CGD. When CGD increases further, the amount of afferent feedback increases. Different Os are differently sensitive to the feedback, and more and more Os begin to change their fixation tendencies toward the intended point of fixation. Because of more Os leaving the group that follows the center of gravity in their fixations, the variability of error increases and the increase in mean error decelerates. At the limit where most of the Os have changed the fixation tendency toward the intended point, the mean error begins to decrease. From Kaufman and Richards's study one could infer that this limit is reached at the latest when the inducing part of the figure exceeds $5 \mathrm{deg}$ of visual angle, but their circle results might indicate that the limit could be under $3 \mathrm{deg}$ of visual angle. Assuming that it is not the figure size per se but a tolerance of the discrepancy between the intended and actual fixation positions that determines how large figures attract center-of-gravity fixations, one could conclude from the circle results that the tolerance limit is under a 1.5-deg CGD. When the inducing figure and CGD grow large enough, the center-of-gravity fixations cease completely, and ail Os begin to fixate the intended point, which results in both small variances and small mean errors. In addition, if the distance between the inducing parts of the figure is small (less than $5 \mathrm{deg}$ ), in case of a very large CGD a tendency to fixate the middle of the test line could occur, producing negative errors.

The discrepancy information from afferent feedback is presumably stored and presumably accumulates with prolonged estimation. Therefore, the amount of illusion decreases as a function of practice. The error should decrease also with age because scanning of environmental objects yields afferent feedback that can be associated with Müller-Lyer patterns. The error should not decrease with prolonged estimation when eye movements are restricted by fixation or short presentations. The illusion should be maximal with such short presentations that are long enough to evoke the center-of-gravity tendency but do not allow afferent feedback from actual eye movements, and the illusion should occur also with stabilized retinal images because stopping of the image should not obliterate the fixation tendency. All these implications are in agreement with empirical data (see Festinger, White, \& Allyn, 1968; Over, 1968; Wohlwill, 1960).

The critical figure variable in the Müller-Lyer illusion should be the CGD measured as visual angle from the vertex of an arrowhead to the geometric center of gravity of the arrowhead. The length of the oblique lines or the length of the test line or the angle between the oblique lines should not be critical, and the error should remain constant with a constant CGD in spite of variation of the angle. Dewar (1967) varied both the oblique length and the angle between the obliques. Computations based on his published results showed that the amount of illusion, if standardized by dividing the error by CGD, was approximately the same for each angle-if any dependence on the angle was present, it was a diminishing error with increasing angle. Erlebacher and Sekuler (1969) varied the angle, keeping the distance between the ends of the obliques constant, which corresponds to a constant CGD. The illusion remained very accurately constant. In the former study the angle between the obliques varied from 30 to $120 \mathrm{deg}$ and in the latter from 20 to $140 \mathrm{deg}$. These results clearly support the model.

In the studies by Dewar and by Erlebacher and Sekuler, the CGD as well as its range of variation were small in terms of the visual angle (less than $1 \mathrm{deg}$ ), and the illusion did not reach its maximum within the range of visual angles tested. Heymans (1896) and Lewis (1909) did not report the viewing distances in their experiments, which makes it impossible to calculate the visual angles, but their ranges of variation were large enough to show that the maxima can occur. There is an obvious need for additional data on the Müller-Lyer illusion as a function of CGD in order to learn where the maxima occur and also if 
Table 1

Lengths of the Obliques (L) and Standard Deviations of the Errors (mm)

\begin{tabular}{rlrrrrrrr}
\hline Angle & CGD & 2.0 & 3.6 & 6.5 & 11.7 & 21.0 & 37.8 \\
\hline \multirow{2}{*}{0 Deg } & L & 4.0 & 7.2 & 13.0 & 23.3 & 42.0 & 75.6 \\
& SD & 2.2 & 3.0 & 2.5 & 2.8 & 3.5 & 2.5 \\
$30 \mathrm{Deg}$ & L & 3.1 & 5.6 & 9.9 & 18.1 & 32.6 & 58.7 \\
& SD & 2.2 & 3.8 & 2.8 & 4.3 & 4.0 & 3.3 \\
$60 \mathrm{Deg}$ & L & 3.5 & 6.2 & 11.0 & 20.2 & 36.4 & 65.5 & 3.4 \\
& SD & 2.5 & 2.9 & 2.9 & 3.9 & 4.9 & 113.4 \\
$120 \mathrm{Deg}$ & L & SD & 6.0 & 10.8 & 19.0 & 35.0 & 63.0 & 3.7 \\
& Sean & SD & 2.0 & 3.8 & 3.7 & 3.6 & 4.0 & 3.1 \\
\hline
\end{tabular}

the illusion is a sole function of CGD over a wider range of visual angles than the range studied so far.

The outgoing-fins case of the conventional Müller-Lyer figure was chosen to be investigated. The model above led to the following expectations guiding the experimental design: (1) The illusion is constant for a given CGD. (2) When the CGD increases, the illusion increases first as a decelerating and monotonic function of CGD, reaches its maximum under $1.5-\mathrm{deg}$ CGD, and diminishes monotonically thereafter. (3) The changes in the variance of the error follow approximately the same course as the error mean.

\section{Method}

Subjects. Twenty-two students, 17 women and 5 men, from an introductory psychology course at the University of Michgian served as Ss.

Stimuli. Twenty-four variants of the outgoing-fins Müller-Lyer figure without the connecting line between the vertices were drawn on $21.5 \times 28 \mathrm{~cm}$ sheets of paper, $3.5 \mathrm{~cm}$ above the sheet middles and parallel to the shorter edges of the sheet. The empty distance between the vertices was $40 \mathrm{~mm}$. The angle between the arms in each arrowhead was $0,30,60$, and $120 \mathrm{deg}$. With the zero angle, a line segment replaces each arrowhead. For every angle, the distance CGD of the center of gravity of the arrowhead from the vertex had values of $2.0,3.6,6.5,11.7$, 21.0 , and $37.8 \mathrm{~mm}$. As visual angles the corresponding values were $.26, .46, .82$, $1.48,2.67$, and $4.80 \mathrm{deg}$. The lengths of the obliques, determined by CGD and arrowhead angles, are presented in Table 1. In addition to the 24 figures explained above, one figure with zero oblique length (two points $40 \mathrm{~mm}$ apart from each other) was drawn for calibrating the illusion measurements.

A line was drawn across every stimulus figure sheet $4 \mathrm{~cm}$ above the lower and shorter edge of the paper. The line was crossed by a $4 \cdot \mathrm{mm}$ reference bar placed $7 \mathrm{~cm}$ to the right from the left edge of the paper. All lines were .3-mm-thick India ink lines.
Procedure. The stimulus figures were photocopied and randomized so that every $S$ received a similar set of the 25 figures but in a different order. The Ss indicated their estimates of the length of the distance between the vertices by marking the estimated length on the line crossing the paper and using the bar as the left boundary. They were asked to erase and correct unsatisfactory estimates after marking. The sheets were presented on a table at about $45-\mathrm{cm}$ eye-figure distance. The shorter edges of the sheets were horizontal with respect to S's visual field.

\section{Results}

The mean errors are presented in Fig. 3. There is a consistent increase in the amount of illusion for all CGDs when the angle becomes larger. In arrowhead figures this is at variance with the earlier results (Heymans, 1896; Lewis, 1909; Dewar, 1967; Erlebacher \& Sekuler, 1969) that indicate either a constant or a slightly decreasing illusion when the angle increases and the CGD remains constant. The reason for this discrepancy is unknown. It may be an interaction effect connected with viewing distance, because in the present experiment the viewing distance was presumably shorter than in any of the other experiments. Nevertheless, the increase in the arrowhead figures is very small relative to the size of the illusion and the large range of variation of the angle.

The result is not in agreement with the first prediction that the error is constant with a constant CGD. Even though the differences among the arrowhead figures may be spurjous, it is clear that the zero-angle figure produced much less illusion than the true arrowhead figures. It is to be noted, however, that the figures with zero angles are qualitatively different from the figures with larger-than-zero angles because they consist of two horizontal line segments. Kaufman and Richards (1969) found that angles were much more potent in attracting fixations than were line segments. It is possible that in line-segment figures a smaller amount of afferent feedback is sufficient to resist the center-of-gravity fixation tendency than in

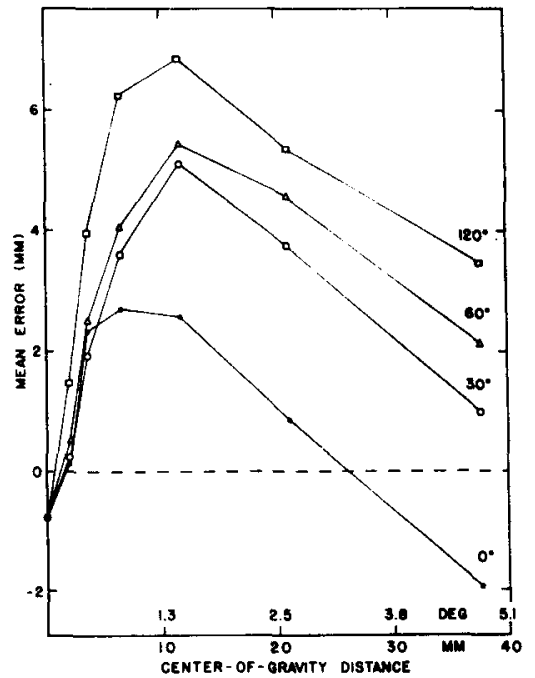

Fig. 3. The Müller-Lyer illusion (30-120 $\mathrm{deg})$ and the line-segment illusion ( $0 \mathrm{deg})$ as a function of the center-of-gravity distance. The parameter is the angle between the obliques.

arrowhead figures. This interpretation is supported by the fact that the curve for the zero angle departs from the ascending course of the error earlier than the other curves. It is quite obvious that the model requires an additional variable for "fixation attractiveness" before it can cope with different figures.

The second prediction was quite successful. The initial increase of illusion is almost linear at every angle and it would be even more linear if the zero reference point (the mean for the control figure of two points) had obtained a little more negative value. The errors form the expected maxima, and the maxima occur approximately at the same center-of-gravity distances in the arrowhead figures. The visual angle of the CGD for the maxima is $1.5 \mathrm{deg}$, which is in agreement with expectations. The value represents a little more than half of the diameter of the fovea, which may be a significant coincidence, because at that CGD the discrepancy between the intended and actual fixation points can become apparent to most of the Os. Assuming that a smaller amount of afferent feedback is sufficient to restrict the error with weaker attractants for fixations, the earlier occurrence of the maximum in the zero-angle figure is not difficult to understand. After the maxima the illusion diminishes monotonically as expected. A negative error, however, occurred only with the longest pair of line segments (a similar negative error is reported by Lewis, 1909).

The standard deviations are presented in Table 1. The lowest row shows the means of the four SDs in order to jllustrate the 
overall course of the variation. The variation grows first and then decreases as expected. The variability maximum occurs, however, later than the error maximum. This could have been expected from the model since it represents what happens if the Ss depart at different times from the course determined by the growing CGD but in spite of the different departure times meet fixating near the vertex when the inducing figure is so large that it does not attract center-of-gravity fixations any longer. The phase shift effect, however, is weak, and an inspection of CGD by $S$ interactions showed that they were too entangled to yield clear-cut additional evidence.

\section{DISCUSSION}

In Experiment 1, tendencies to rectilinear and horizontal or vertical eye movements were inferred from eye-movement records of Stroud (Whitteridge, 1960), Dodge (1907), Stratton (1902), and Yarbus (1967). From such movement tendencies underestimation of curvature and perceptual inclination of continuations of arcs were predicted. In the experiment, Ss misperceived the location of intersection of two arc segments as expected, and $92 \%$ of the total variance of the error means was explained by the two factors. There was also a decrement in the error in the course of estimation, as would be predicted from afferent feedback.

In Experiment 2, a simple model based on the tendency to fixate toward the center of gravity of a figure was proposed to explain Müller-Lyer type of illusion. This fixation tendency has been reported by Kaulman and Richards (1969), and Richards and Kaufman (1969). The results of the experiment with arrowhead and line-segment figures gave moderate support to the model. The course of the error as a function of the center-of-gravity distance had the expected form, and variations of standard deviations were in agreement with the model. However, the error was slightly smaller with smaller angles between the obliques and considerably smaller in line-segment figures than in arrowhead figures. This result can be understood, at least in part, by the fact that different figures are not equipotent as attractants for fixations (Kaufman \& Richards, 1969). Because only little is known about this variable, it was not taken into account in the model in its present form, and therefore the model incorrectly predicted a constant error for a constant CGD. The model requires an additional parameter for fixation attractiveness.

The overall success of the predictions suggests that it is possible to infer movement tendencies from records of actual movements and utilize the abstracted movement tendencies for predicting how a geometric form is perceived by Os whose background experiences are not known. Thus, it seems possible to extend the notion that tendency to movement or efferent readiness influences perception to explain phenomena of actual perception and not only perceptual modifications during an experiment. Even in its present elementary form the notion produces quite strong predictions and has great potential for further development. At least, the notion seems sufficient to explain the results of the present experiments as well as a large number of earlier results dealing with temporal, experiential, and developmental variables in the Müller-Lyer illusion, giving an integrated account for both the occurrence of the illusion and its decrement with prolonged practice.

These results, of course, are only indirect evidence for the notion that tendencies to movement influence perception, and, in fact, direct testing of the notion is very difficult to accomplish in practice. In principle, it is possible to test the notion directly, for the notion must be discarded if it is possible to show, for example, that a change in perception of form is not preceded by a change in relevant tendencies to movement as inferred from free movements. The notion becomes very doubtful also if it is possible to find changes in movement tendencies that are not followed by respective perceptual changes, but this does not necessarily show that the notion is incorrect. Another weakness of the notion is that it may be impossible to show that the concept of tendency to movement is necessary for explaining any perceptual results. Tendency to movement is a theoretical construct inferred from the occurrence of actual movements, and the tendency is assumed to exist and to be effective also in absence of movements if explicit movements are not allowed, as with short stimulus presentations, stopped images, and fixation, provided that the tendency in question is meaningful for the perceptual task given to the $O$. In many interesting cases it is not possible to identify the nonexistence of a given tendency to movement.

The main justification of the concept of tendency to movement is in the fact that it is a convenient and economical tool for integrating a large set of experimental results. The integration of the illusion results discussed in this study is not possible in terms of explicit eye movements since the Müller-Lyer illusion occurs in absence of eye movements.
Explicit eye movements that provide an 0 with information about his errors when scanning an illusion figure seem to be a necessary condition only for the decrement of the illusion. It is also difficult to see how the results could be integrated using the traditional explanations of geometrical illusions.

Over (1968) discusses seven theories of geometrical illusions that attempt to explain illusions in terms of retinal induction, cortical satiation, directional (explicit) eye movements, inappropriate constancy scaling, lateral inhibition, centration, and contextual learning. He rejects the first four explanations because they predict absence of illusion under conditions where illusion occurs. Of these four, the eye-movement explanation is one of the earlier, unsuccessful forms of the present explanation, and the other three explanations in terms of retinal induction, cortical satiation, and inappropriate constancy scaling cannot explain the intersection illusion of Fig. 1 or the experiential effects on the Muiller-Lyer illusion.

The lateral-inhibition explanation does not apply to the present results because in both experiments the possibly interacting contours were too far from each other in order to produce the necessary inhibition effects. In addition, lateral inhibition should produce repulsion rather than attraction effects, but attraction is typical of Müller-Lyer-type illusions. The contextual learning explanation is hardly applicable to the intersection illusion of Experiment 1, and so far as the decrement of the Müller-Lyer illusion is explained by assuming that with prolonged inspection Os attend less to nonrelevant components of the figure, the present model explains why this happens and why the nonrelevant components have been attended to in the first place. Piaget's centration theory (see Piaget, 1969) has been very successful in explaining the error functions in various illusions, and it certainly can account for the Müller-Lyer data of the present experiment as well as of the related experiments referred to in this study. However, the explanation of the Müller-Lyer results in terms of tendency to center-of-gravity fixations is considerably simpler than in terms of centrations. On the other hand, Piaget's theory is probably not adequate for explaining the results of Experiment 1 because in the intersection illusion the identification of relevant size inequalities is most difficult. The identification of attention-evoking features in illusion figures is the general difficulty with the centration theory, and therefore the application of the theory sometimes seems quite arbitrary. 


\section{REFERENCES}

DAY, R. H. The effects of repeated trials and prolonged fixation on error in the Müller-Lyer figure. Psychological Monographs, 1962, 76(14, Whole No. 533).

DEWAR, R. E. Stimulus determinants of the magnitude of the Müller-Lyer illusion. Perceptual \& Motor Skills, 1967, 24, 708-710.

DODGE, R. An experimental study of visual fixation. Psychological Monographs, 1907, 8 (Whole No. 35).

ERLEBACHER, A., \& SEKULER, R. Explanation of the Müller-Lyer illusion: Confusion theory examined, Journal of Experimental Psychology, 1969, 80, 462-467.

FEHRER, E., \& RAAB, D. Reaction time to stimuli masked by metacontrast. Journal of Experimental Psychology, 1962,63, 143-147.

FESTINGER, L., BURNHAM, C. A., ONO, H., \& BAMBER, D, Efference and the conscious experience of perception. Journal of Experimental Psychology Monograph, 1967, 74 (Whole No. 637).

FESTINGER, L., WHITE, C. W., \& ALLYN, M. $R$. Eye movements and decrement in the Müller-Lyer illusion. Perception \& Psychophysics, 1968, 3, 376-382.

GROVITZ, H. F., \& DAVES, W. Tendencics to eye movement and perceptual accuracy. Journal of Experimental Psychology, 1962 , 63, 495-498.

HEYMANS, G. Quantitative Untersuchungen über das "optische Paradoxon." Zeitschrift für Psychologie, 1896, 9, 221-255.

HOFMANN, F. B., \& BIELSCHOWSKY, A. Über die Einstellung der scheinbaren Horizontalen und Vertikalen bei Betrachtung eines von schrägen Konturen erfullten Gesichtsfeldes. Archiv tur die gesamte Physiologie, 1909, 126, 453-475.

KAHNEMAN, D. Method, findings, and theory in studies of visual masking. Psychological Bulletin, 1968, 70, 404-425.

KAUFMAN, L., \& RICHARDS, W. Spontaneous fixation tendencies for visual forms. Perception \& Psychophysics, 1969, 5, 85-88.

LEWIS, E. O. Confluxion and contrast in the Müller-Lyer illusion. British Journal of Psychology, 1909, 3, 21-41.

MAYZNER, M. S., TRESSELT, M. E., \& HELFER, M. S. A provisional model of visual information processing with sequential inputs.
Psychonomic Monograph Supplement, 1967, 2, 91-108 (Whole No. 23).

Mclaughlin, S. C., DeSISTO, M. J., \& KELLY, M. J., JR. Comment on "Eye movements and decrement in the Müller-Lyer illusion." Perception \& Psychophysics, 1969, $5,288$.

MCLAUGHLIN, S. C., KELLY, M. J., JR., ANDERSON, R. E., \& WENZ, T. G Localization of a peripheral target during parametric adjustment of saccadic eye movements. Perception \& Psychophysics, $1968,4,45-48$.

MOUNTJOY, P. T. Effects of self-instruction information and misinformation upon decrement to the Mueller-Lyer figure. Psychological Record, 1965, 15, 7-14.

OBONAI, T. Experimentelle Untersuchungen über den Aufbau des Sehraumes. Archiv für die gesamtc Psychologie, 1931, 82, 308-328.

OVER, R. Explanations of geometrical illusions. Psychological Bulletin, 1968, 70, 545-562.

PIAGET, J. The mechanisms of perception. New York: Basic Books, 1969.

PIAGET, J., \& BANG, V. L'enregistrement des mouvements oculaires en jeu chez l'adulte dans la comparaison verticales, horizontales ou obliques et dans les perceptions de la figure en équerre. Archives de Psychologie, 1961, 38, 89-141.

RICHARDS, W., \& KAUFMAN, L. "Center-of-gravity" tendencies for fixations and flow patterns. Perception \& Psychophysics, $1969,5,81-84$

STRATTON, G. M. Eye movements and the aesthetics of visual form. Philosophische Studien, 1902, 20, 336-359.

WEINTRAUB, D. J., \& VIRSU, V. The misperception of angles: Estimating the vertex of converging line segments. Perception \& Psychophysics, 1971, 9, 5-8.

WHIIERIDGE, D. Central control of eye movements. In J. Field, H. W. Magoun, and V. E. Hall (Eds.), Handbook of physiology, Section 1: Neurophysiology. Vol. 3. Washington, D.C: American Physiological Association, 1960. Pp. 1595-1668.

WOHLWILL, J. F. Developmental studies of perception. Psychological Bulletin, 1960, 57, 249.290.

YARBUS, A. L. Eye movements and vision. New York: Plenum Press, 1967.

\section{NOTES}

1. If one assumes that rectilinear movement tendencies influence perception, it is necessary to postulate some kind of perceptual leveling process or "Prägnanz tendency" to account for the fact that the shape of a circle is not distorted in perception. The existence of this process is quite plausible, however, for examples of leveling phenomena abound in Gestalt literature, the best known of which are the subjective completion of the blind spot and the disappearance of brightness contrast in Koffka's ring.

2. Among other possible confounding factors, misperception of the angle between the two line segments cannot be separated from the results of Obonai (1931) and of Weintraub and Virsu (1971). Therefore, one cannot expect that Function 1 , approximating only the perceptual tilt toward the cardinal axes, could fit perfectly to those results. However, in order to illustrate the goodness of fit of Function 1 to the combined data of the two experiments by Weintraub and Virsu, the least-square estimate of $b_{1}$ was calculated. The resulting function, $B=-1.46 \sin 4 A$, explained $49 \%$ of the variance of the error means of the two experiments showing that, in spite of the possible impurities of the data, Function 1 gives a useful one-parameter description of the complicated error functions. Function 1 would fit better to the results of Hofmann and Bielschowsky (1909), but calculations on them were not performed because of the idiographic nature of their data.

3. Virsu, V. Underestimation of curvature, and task dependence in visual perception of form. In preparation.

4. Richards and Kaufman (1969) reported that there is also a strong upward-looking tendency for many Os. This movement tendency may be a basic factor in the overestimation of vertical extents relative to horizontal ones. In fact, Piaget and Bang (1961) found that there was a tendency to fixate the summit of the vertical line and the middle of the horizontal line in the horizontal-vertical illusion-figure. Obviously, a vertical line evokes a tendency to perform longer eye movements than a horizontal line, and the illusion can be a direct consequence of this movement tendency.

(Accepted for publication May 28, 1970.) 